\section{The Use of Allograft Bone Morphogenetic Protein in Foot and Ankle Arthrodesis}

\author{
J George DeVries* and Brandon Scharer \\ Orthopedics and Sports Medicine, BayCare Clinic, Riverside Dr, Green \\ Bay, WI, USA
}

\begin{abstract}
Bone grafting procedures continue to be an important and common surgery performed in the foot and ankle. Alternatives to autogenously harvested are continuously discovered and researched. A novel allogeneic morphogenetic protein harvesting process has shown promise in spine fusion surgery. A retrospective chart and radiographic review of the first 10 consecutive patients to utilize this graft was undertaken to determine initial outcomes and safety profile. Ultimately fusion was obtained in $90 \%$ (9 out of 10 ) of patients, and in $95.7 \%$ (22 out of 23 ) of arthrodesis sites. Weight bearing occurred at $59.9 \pm 20.6$ days, and radiographic union took place at $78.5 \pm 42.4$ days. Based on these initial results the graft appears safe and effective. Because of several inherent advantages of the graft, further study should be undertaken.
\end{abstract}

Keywords: BMP; Bone graft; Nonunion; Reconstruction; Revision

\section{Level of Clinical Evidence}

4 , retrospective case series

\section{Introduction}

Bone grafting in foot and ankle surgery for fractures, osteotomies, and arthrodesis is a common practice [1]. Due to concerns over autogenous donor site morbidity [2,3] graft substitutes have been developed. In foot and ankle surgery, two main autograft alternatives have been put forward as alternatives for high risk procedures, recombinant human Bone Morphogenetic Protein (BMP) and allograft viable Mesenchymal Stem Cells (MSC). BMPs are powerful osteoinductive graft materials, and have demonstrated clinical effectiveness in nonunions, segmental defects, and long bone fractures [4-7]. Evaluation of its application (off-label) in foot and ankle surgery has generally been favorable [8-12]. On the other hand, MSCs have also demonstrated effectiveness in surgery [13]. Commercially available MSCs approved for the use in foot and ankle arthrodesis provide osteogenic, osteoconductive, and osteoinductive

*Corresponding author: J George DeVries, Orthopedics and Sports Medicine BayCare Clinic, Riverside Dr, Green Bay, WI, USA, Tel: +1 9202885555; Fax: +1 9202885550; E-mail: jdevries@baycare.net

Citation: DeVries JG, Scharer B (2016) The Use of Allograft Bone Morphogenetic Protein in Foot and Ankle Arthrodesis. J Orthop Res Physiother 2: 023.

Received: 06, March 2016; Accepted: 02, May 2016; Published: 16, May 2016 properties, and have been recommended in various applications [14-16].

A novel processing method has provided a way to obtain Allogeneic Morphogenetic Protein (AMP) from allograft and its native bone marrow. This process has been purported to allow for higher concentrations of growth factors (such as BMP-2, BMP-7, Transforming Growth Factor (TGF)- $\beta 1$ ) to bond to the scaffold and be delivered to the operative site [17]. AMP (OsteoAMP, Bioventus LLC, Durham, NC, USA) is available in compressible sponge, putty, and granule formats, and can be combined with Bone Marrow Aspirate (BMA). Also, it has been evaluated in several clinical trials in the spine. Fusion rates in spinal surgery were not affected by graft variability, and specifically looked at donor age, donor sex, age of graft from harvest to implantation, and aseptic or terminal irradiation [18], indicating predictable quality of the product. It was also evaluated in cervical spine surgery, and demonstrated high fusion rates [19]. A study of 321 patients undergoing various spine fusion operations was undertaken to compare AMP to BMP. The 226 patients in the AMP arm compared very well to the 95 patients in the BMP arm in terms of fusion rate, time to fusion, and complications [20].

AMP is approved for use in situations where an autograft is appropriate and can be used for repair, replacement or reconstruction of skeletal defects, and has on-label use in the foot and ankle. The various growth factors provide osteoinduction, the various formats provide osteoconduction, and when combined with BMA provides autogenous osteogenic cells [21], thus replicating all three pillars of bone healing. This study was undertaken to evaluate initial safety and efficacy of AMP in foot and ankle surgery. The hypothesis was that AMP will have outcomes in foot and ankle surgery comparable to other products with no unexpected complications. The primary objective was to evaluate safety of its use in foot and ankle surgery, with the secondary objective of time and rate of union.

\section{Patients and Methods}

Institutional review board approval (15-50E) was obtained for the present study. A retrospective chart and radiographic review was performed on the first ten patients that underwent foot and ankle utilizing AMP (OsteoAMP) from November 2014 to February 2015. Patients were identified by review of cases utilizing AMP. Inclusion criteria included all patients that underwent surgical intervention and utilized AMP. Exclusion criteria included incomplete patient information, or lack of use of AMP. Indication for use of AMP was surgeon dependent, but was selected in high risk patients. This included revision surgery, smoking, medical co-morbidities, advanced age, and obesity. Surgery was performed using standard surgical technique dependent on the specific surgical indication. All surgeries were performed by one of the two authors, fellowship trained foot and ankle surgeons. Each surgeon contributed 5 patients to the current study.

Although surgical indications, approach, and fixation were different for the various cases, certain standard techniques were utilized. In all revision operations, the same incision was reused and all impeding hardware was removed. All cases focused on bone fusion, 
whether primary arthrodesis, revision of arthrodesis nonunion, or fracture nonunion. In all cases, the proposed fusion site was exposed, and any remaining articular surface was removed past subchondral bone. For nonunion, this included debridement of fibrotic material to raw, bleeding bone. The area was then fenestrated with small drill or pin. Once the arthrodesis site was prepared, it was packed with AMP and any other grafting material used. Fixation was specific to the anatomic site, but in all cases was meant to provide compression and stability. Forefoot cases were allowed to weight bear in a protective boot at 2 weeks, while all midfoot, hindfoot, and ankle cases were restricted to a non weight bearing splint and then fiberglass cast for 6-8 weeks (Figure 1).

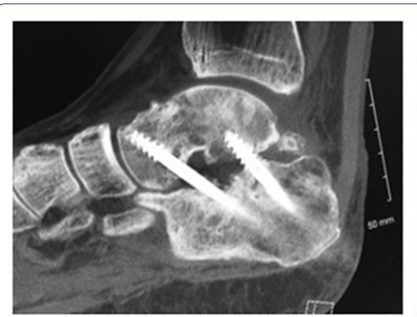

Figure $1 \mathrm{~A}$

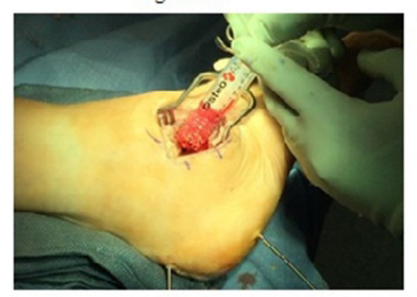

Figure 1C

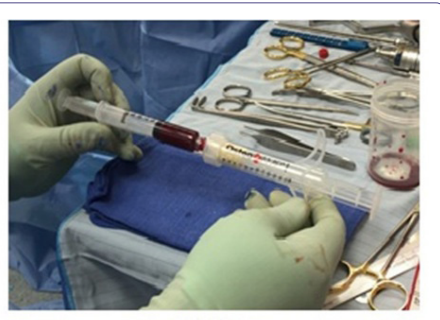

Figure 1B

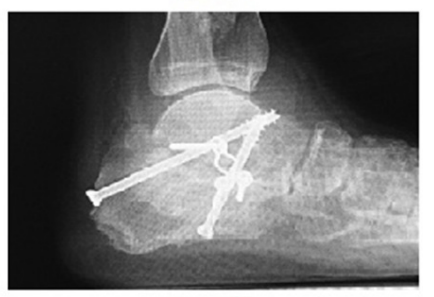

Figure 1D
Figure 1: A Shows a CT scan revealing nonunion at subtalar joint with hardware failure. $B$ is preparation of $5 \mathrm{~mL}$ of allogeneic bone morphogenetic protein granules being mixed with $6 \mathrm{~mL}$ of bone marrow aspirate. $C$ shows delivery of grafting materials into the nonunion site. $D$ is a weight bearing lateral plain radiograph at 3 months post-operatively showing solid arthodesis with alternative hardware placement.

Medical chart review recorded age, gender, ethnicity, past medical history, social history, indication, time to weight bearing, and complications. Radiographic review was undertaken to determine time to radiographic fusion. This was defined as radiographic evidence of bridging bone, with lack of motion or hardware complications. This was performed by the authors. Since no comparisons are to be made, only simple statistical analysis was performed. Patient demographics will be presented as mean with standard deviation and ranges of continuous variables such as age, time to weight bearing, and time to radiographic union. In order for the surgery to be classified as union, bridging bone needed to be appreciated on plain films, and the patient had to be clinically fused with no further swelling or pain. This was assessed by the operating surgeon. Categorical variables such as gender, ethnicity, past medical history and social history, and complications will be represented as percentages of the group.

\section{Results}

The study population included 5 females (50\%), and 5 males (50\%), with an average age of $57.3 \pm 13.3$ years (range: $29-80$ years). All patients were Caucasian. Patients had a variety of medical co-morbidities, including Diabetes Mellitus (DM), chronic kidney disease, and a variety of mental disorders (Table 1). Social risk factors were recorded, and demonstrated that 4 patients (40\%) never used tobacco and 5 patients (50\%) did not use any alcohol. Of the patients that smoked, only 1 was an active smoker, and the others had quit an average of $18.0 \pm 8.8 \mathrm{yrs}$ (range: 8 -32 years) prior to the studied operation. The one patient that was an active smoker also drank 15 drinks per week, and used marijuana daily, and was the only patient that related illegal drug use. Patients were followed an average of $167.6 \pm 21.8$ days (range: 125 - 188 days) from surgery.

\begin{tabular}{|c|c|}
\hline Disease & Prevalence $(\mathbf{n = 1 0})$ \\
\hline Hypertension & $50 \%$ \\
\hline Diabetes Mellitus & $40 \%$ \\
\hline Mental Disorder* & $30 \%$ \\
\hline Chronic Kidney Disease & $10 \%$ \\
\hline Anemia & $10 \%$ \\
\hline Gout & $10 \%$ \\
\hline \multicolumn{2}{|c|}{ Table 1: Medical Co-morbidities. } \\
\hline
\end{tabular}

Mental disorder includes one or more of the following: Attention deficit disorder, Obsessive compulsive disorder, Anxiety, Depression

The patients underwent a variety of surgical treatments. Nonunion of previous arthrodesis or of fracture or revision of failed prior surgery was the indication for 5 patients (50\%) (Figures 2 and 3). A total of 23 fusion sites were represented in the study population. Adjunctive procedures were performed in 7 patients (70\%). Autogenous grafting was utilized in 3 patients $(30 \%)$ and BMA was used in 5 patients (50\%). A detailed list of surgical variables is listed in table 2 .

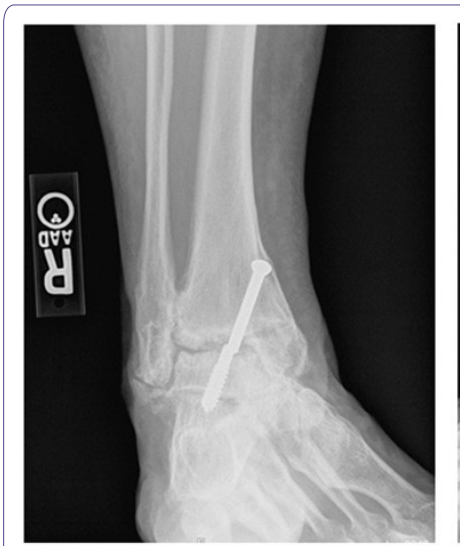

Figure $2 \mathrm{~A}$

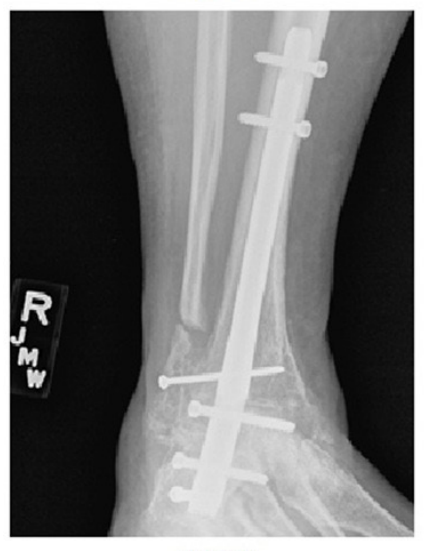

Figure 2C

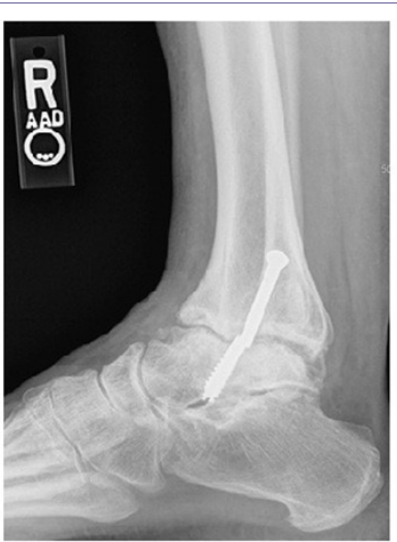

Figure 2B

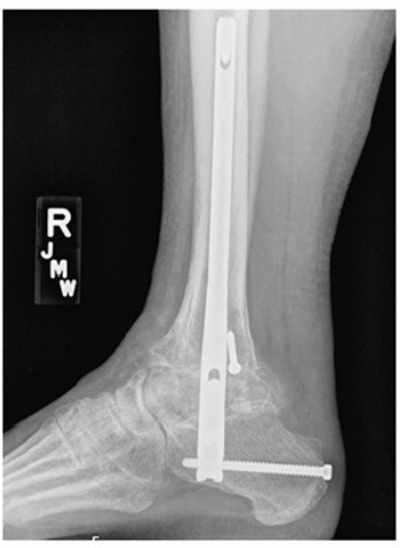

Figure 2D
Figure 2: A (mortise) and B (lateral) are pre-operative images of patient 3 demonstrating ankle nonunion with anterior translation, subtalar joint arthritis, broken hardware. C (mortise) and D (lateral) are post-operative images demonstrating solid union at the ankle and subtalar joints, as well as the fibula onlay site. 


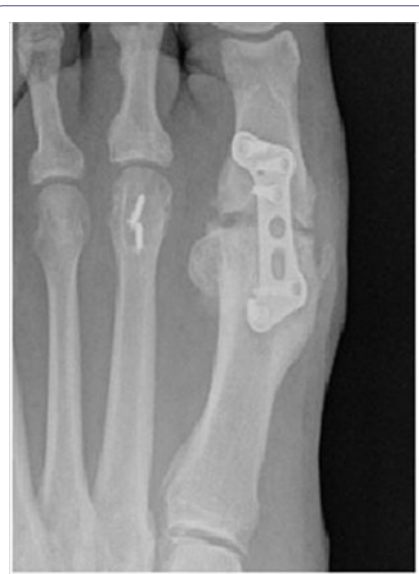

Figure $3 \mathrm{~A}$

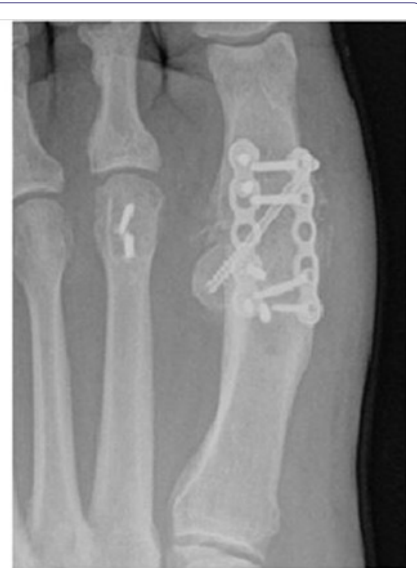

Figure 3B
Figure 3: Patient 1 shows pre-operative nonunion of the 1 st metatarsal phalangeal joint with broken proximal screw (A) and post-operative arthrodesis (B).

Ultimately, 22 out of 23 fusion sites (95.7\%) in 9 patients (90\%) went on to solid arthrodesis. The average time to weight bearing was $59.9 \pm 20.6$ days (range: $14-78$ days). Average time to radiographic healing was $78.5 \pm 42.4$ days (range: 42 - 187 days). Overall, 2 patients (20\%) experienced complications. This included 1 wound dehiscence that was treated with local wound care and oral antibiotics, and 1 nonunion of the medial malleolus. The nonunion was stable and the patient has not undergone revision surgery.

\section{Discussion}

Bone grafting procedures are frequently performed in orthopedic surgery, with more than 500,000 bone grafting procedures performed each year in the United States alone [22]. Historically, autogenous bone graft has been considered the "gold standard", and typically has been discussed as graft taken from the iliac crest. This has been described in spine surgery $[23,24]$ and also specifically evaluated in foot and ankle surgery [25-27]. However, because of complications associated with the harvest of the autogenous graft, alternatives to the iliac crest have proliferated [28-30]. This has led to development of over 200 different commercially available bone graft substitutes, some of which can be very expensive and most with little or no high level evidence to support their use [31]. Research continues to find the ideal autogenous bone graft alternative. Ideally, this will be something that is effective, safe, readily available, non-morbid and relatively inexpensive.

An article by Roh et al., in 2013 was published comparing recombinant human BMP (rhBMP-2, Infuse, Medtronic Inc., Fridley, $\mathrm{MN}$, USA) to AMP (OsteoAMP) in lumbar interbody fusion procedures [20]. This was a multicenter study comparing the fusion rate of the two products, as well as the cost associated with them. A total of 321 consecutive patients were evaluated over 5 years, 95 in the BMP group and 226 in the AMP group. The study reported universally favorable findings of AMP versus BMP, including time to fusion, percentage fusion, and complications. In addition, the AMP group also reported approximately $80 \%$ lower costs as well (about $\$ 650$ for AMP vs $\$ 2500$ for BMP). Several concerns are raised about the article. Statistical analysis of the findings between the groups was not consistently reported, and there was a potential commercial bias [32]. Even with these limitations, the results were impressive and lead to the current investigation.
The AMP used in this investigation not only has favorable clinical studies [18-20], but internal data and analysis also compares favorably to competing products. It shows high levels of BMP-2, BMP-7, and TGF- $\beta 1$, and others in the sponge product [17]. In addition to the clinical and laboratory data, the product is also practical and versatile in the surgical setting and has excellent handling abilities. It is available in a compressible sponge, and has higher concentrations of BMP-2 than another commercially available compressible sponge product (Bacterin Osteosponge; Bacterin International Holdings, Inc., Belgrade, MT, USA) [17]. The authors in the present study utilized the putty or the granule forms and mixed with BMA in $50 \%$ of the cases. Bone marrow aspirate can quickly, easily, and safely be harvested from lower extremity sites, and although there are fewer colony-forming units in lower extremity sites compared to the iliac crest, only a minimal concentration of cells may be needed to achieve clinical fusion [33-35].

High risk foot and ankle fusions are often augmented with some biologic adjunct. Two options that have been recommended are recombinant human BMP and MSC. After extensive study in long bone fractures, segmental defects, and nonunions, recombinant human BMP was explicitly studied in the foot and ankle. A study published in 2009 by Schuberth et al., performed a multicenter evaluation of recombinant human BMP in foot and ankle surgery. Overall, out of 38 procedures in 35 patients, the incidence of successful healing was $84.2 \%$ ( 32 procedures), and multiple statistical analyses teased out predictors of success [8]. Another study in 2009 evaluated 69 patients, but broke the results down to 112 fusion sites augmented with recombinant human BMP. The authors reported a $96 \%$ fusion rate (108 sites), and this was confirmed with CT scan. The patient population consisted of $64 \%$ smokers, $19 \%$ diabetic patients, $68 \%$ high energy trauma, and 22\% Avascular Necrosis (AVN) of the talus. In addition to the excellent fusion rate, low complication rates were reported as well [11]. DeVries et al., in contrast reported a 69.6\% (16 of 23 patients) rate of arthrodesis for revision nonunion cases treated with intramedullary tibiotalocalcaneal nail. No clear benefit was demonstrated with the addition of recombinant human BMP, although again no specific adverse effects of the BMP were seen [12].

Similarly, evaluation of MSCs has been undertaken in the foot and ankle. Rush et al., published several studies looking into the use of MSCs, including a retrospective review of 23 patients in 2009. Here the authors found an overall union rate of $91.3 \%$ (21 patients), and observed new bone formation in all patients at the level of implantation. No graft specific complications were found [14]. Scott and Hyer also evaluated this graft in 20 patients in 2013. All patients were undergoing primary arthrodesis, but were classified as "high-risk" based on smoking status, high Body Mass Index (BMI), or presences of diabetes. The authors presented a 90\% (18 patients) union rate, with 2 patients resulting in nonunion that required additional surgery. Again, no graft specific complications were noted [15].

The current study demonstrates that AMP compares very well to both BMP and MSC. There was successful arthrodesis in $90 \%$ (9 out of 10) patients, and this included 95.7\% (22 out of 23) total arthrodesis sites. The graft also demonstrated new bone formation in these patients (Figure 4). This included "high-risk" patients including revision surgery, diabetics, advanced age, and smokers. AMP has some notable advantages over these other adjuncts. Recombinant human BMP (Infuse) is indicated for spinal fusion and open tibia fractures, and is not specifically intended for use in the foot and ankle, and has been reported to have some adverse events in the 


\begin{tabular}{|c|c|c|c|c|c|c|c|}
\hline Patient & Indication & Side & Procedure & Quantity & Type & Adjunctive Procedures & $\begin{array}{c}\text { Number of } \\
\text { Arthrodesis Sites }\end{array}$ \\
\hline 1 & 1st MTP nonunion & Left & 1st MTP nonunion revision & $1 \mathrm{cc}$ & Putty & HWR, BMA & 1 \\
\hline 2 & Ankle malunion, STJ nonunion & Right & $\begin{array}{l}\text { Ankle malunion revision, STJ } \\
\text { nonunion revision }\end{array}$ & $5 \mathrm{cc}$ & Granules & HWR, fibular grafting & 2 \\
\hline 3 & Ankle nonunion, STJ DJD & Right & $\begin{array}{l}\text { Ankle nonunion revision, STJ } \\
\text { arthrodesis, fibula onlay graft }\end{array}$ & $5 \mathrm{cc}$ & Granules & TAL, HWR, BMA & 3 \\
\hline 4 & Ankle DJD & Right & Ankle fusion & $5 \mathrm{cc}$ & Granules & & 1 \\
\hline 5 & $\begin{array}{c}\text { Medial and lateral malleolar } \\
\text { nonunion }\end{array}$ & Left & Bimalleolar nonunion ORIF & $5 \mathrm{cc}$ & Putty & BMA & 2 \\
\hline 6 & PTTD stage 3, hindfoot DJD & Right & Triple arthrodesis & $5 \mathrm{cc}$ & Putty & TAL, BMA & 3 \\
\hline 7 & Midfoot DJD & Left & 1-2 TMT and 1-2 NC arthrodesis & $5 \mathrm{cc}$ & Putty & GSR, distal tibial graft, BMA & 4 \\
\hline 8 & Midfoot DJD & Left & 1st TMT and 1-2 NC arthrodesis & $5 \mathrm{cc}$ & Putty & GSR & 3 \\
\hline 9 & Failed flatfoot reconstruction & Left & TN and STJ arthrodesis & $10 \mathrm{cc}$ & Granules & GSR & 2 \\
\hline 10 & $\begin{array}{l}\text { Ankle Charcot neuro-arthropathy, } \\
\text { talar AVN }\end{array}$ & Right & Tibio-talocalcaneal arthrodesis & $10 \mathrm{cc}$ & Granules & & 2 \\
\hline
\end{tabular}

Table 2: Detailed surgical data.

AVN: Avascular Necrosis; BMA: Bone Marrow Aspirate; DJD: Degenerative Joint Disease; GSR: Gastrocnemius Recession; HWR: Hardware Removal; MTP: Metatarsal Phalangeal Joint; NC: Naviculocuneoform; ORIF: Open Reduction, Internal Fixation; PTTD: Posterior Tibialis Tendon Dysfunction; STJ: Subtalar Joint; TAL: Tendo Achilles Lengthening; TMT: Tarsometatarsal; TN: Talonavicular

spine and an increase in cancer incidence of 1.8-3\% [36]. It also has poor handling characteristics and does not provide any space-filling properties. The viable cells in MSC are a benefit, but also have drawbacks. The product must be stored in a freezer at $-70^{\circ} \mathrm{C}$ to $-80^{\circ} \mathrm{C}$ and has a shelf life of 3 months. Careful thawing of the product has to be undertaken to ensure cell viability, and must be used within 2 hours of thawing [16]. The AMP product provides high levels of BMP-2 as well as a variety of other growth factors. It provides a scaffold and space-filling properties, and when mixed with autogenous BMA can provide all three properties of bone healing. It is freeze dried and can be stored at room temperature for 5 years. Finally, the AMP used in this study (OsteoAMP) is substantially less expensive than other products at the authors institution. A package of $5 \mathrm{cc}$ granules is priced at $\$ 1755.00$, compared to medium Infuse $e^{\infty}$ priced at $\$ 4893.00$ and 5 cc of Trinity Evolution (Orthofix, Verona, Italy) priced at $\$ 2259.00$. Although a cost comparative analysis is beyond the scope of this paper, cost advantages were cited in spine literature [20].

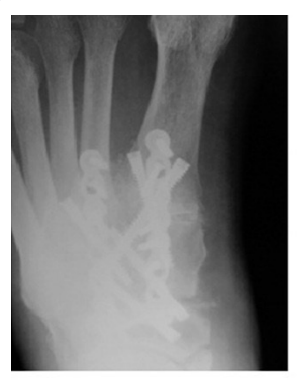

Figure 4A

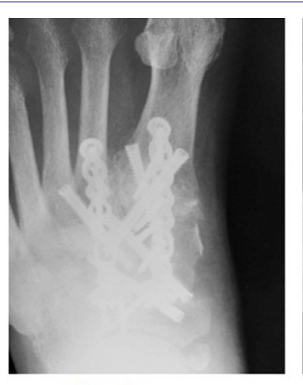

Figure 4B

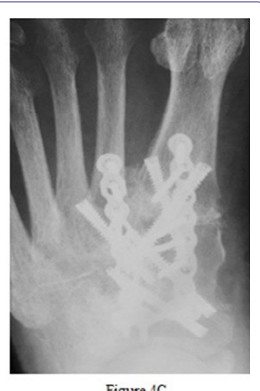

Figure 4C
Figure 4: New bone growth is demonstrated in patient 7. AP image two weeks post-operative (A) shows open joints at the 1st tarsometatarsal and medial naviculocuneiform. AP image at 48 days post-operative (B) shows solid arthrodesis at those joints. AP image at 139 days post-operative (C) demonstrates new bone growth at the $1 \mathrm{st}$ interspace between the $1 \mathrm{st}$ and 2nd metatarsal base.

The single incidence of nonunion was present in a patient that smoked tobacco and marijuana daily, was a heavy drinker (15 drinks/week), and was non-compliant with his non-weight bearing status immediately after surgery. He had presented 3 months after displaced trimalleolar fracture with nonunion at all 3 fracture sites, and stated that his pain had been well controlled throughout, likely due to his daily marijuana use, which he admitted to increasing after the injury. This also was likely a determining factor in his recovery as he stated he had very little pain and did not require pain medications after surgery, despite early weight bearing and having an obvious medial malleolar nonunion. And even with this, he did go onto a solid union of his fibular nonunion.

This retrospective study was intended to be an early analysis of the first patients in which the AMP product was utilized. This was done purposely because of the limited amounts of available literature describing this product, and an uncertainty in the expected outcomes in foot and ankle surgery. There are several important limitations that were accepted at the outset of this report. There is no comparative group, there is a small sample size, and the follow up is short. Without a control or comparative group it is impossible to determine how many of these patients would have healed simply with the revision operation. There is no patient functional data or high level statistical analysis. In addition, several patients had autogenous grafting used in conjunction to the AMP which could have influenced the results. The inclusion criteria was at the total discretion of the operating surgeon as this represented the first cases in which AMP was used, and thus could introduce bias. Bias could also be introduced in the radiographic determination of union. CT scan would have been ideal, but as an early look at these patients, the cost and additional radiation was deemed unnecessary for the purposes of the paper. The aim of the report was to explore the use of a novel allograft bone graft alternative, and establish whether this seemed to be safe and effective for these high risk operations. Based on these early findings, a more robust clinical trial is planned, and needs to be undertaken to delineate this products place in the surgeons' armamentarium.

In conclusion, AMP is found to have similar outcomes as would be expected for other studied bone graft materials. It is difficult to draw firm conclusions based on this early evaluation due to the stated limitations. However, no graft specific adverse reactions were identified. There are several practical advantages to the current product, and further study was deemed warranted. Based on these findings, the authors are collecting further data to address several of this limitations. Advanced biologics such as the one explored in this work continue to show great promise and utility in foot and ankle 
surgery, especially in the face of higher risk populations and procedures.

\section{Financial Disclosure}

Dr. DeVries is a consultant with Bioventus, owner of OsteoAMP

\section{References}

1. DiDomenico LA, Thomas ZM (2015) Osteobiologics in foot and ankle surgery. Clin Podiatr Med Surg 32: 1-19.

2. DeOrio JK, Farber DC (2005) Morbidity associated with anterior iliac crest bone grafting in foot and ankle surgery. Foot Ankle Int 26: 147-151.

3. Geideman W, Early JS, Brodsky J (2004) Clinical results of harvesting autogenous cancellous graft from the ipsilateral proximal tibia for use in foot and ankle surgery. Foot Ankle Int 25: 451-455

4. Termaat MF, Den Boer FC, Bakker FC, Patka P, Haarman HJ (2005) Bone morphogenetic proteins. Development and clinical efficacy in the treatment of fractures and bone defects. J Bone Joint Surg Am 87: 1367-1378.

5. Yasko AW, Lane JM, Fellinger EJ, Rosen V, Wozney JM, et al. (1992) The healing of segmental bone defects, induced by recombinant human Bone Morphogenetic Protein (rhBMP-2). A radiographic, histological, and biomechanical study in rats. J Bone Joint Surg Am 74: 659-670.

6. Friedlaender GE, Perry CR, Cole JD, Cook SD, Cierny G, et al. (2001) Osteogenic protein-1 (bone morphogenetic protein-7) in the treatment of tibial nonunions. J Bone Joint Surg Am 83-83A: 151-158.

7. Govender S, Csimma C, Genant HK, Valentin-Opran A, Amit Y, et al. (2002) Recombinant human bone morphogenetic protein-2 for treatment of open tibial fractures: a prospective, controlled, randomized study of four hundred and fifty patients. J Bone Joint Surg Am 84-A: 2123-2134

8. Schuberth JM, DiDomenico LA, Mendicino RW (2009) The utility and effectiveness of bone morphogenetic protein in foot and ankle surgery. $J$ Foot Ankle Surg 48: 309-314.

9. Kain MS, Einhorn TA (2005) Recombinant human bone morphogenetic proteins in the treatment of fractures. Foot Ankle Clin 10: 639-650.

10. Bibbo C, Patel DV, Haskell MD (2009) Recombinant Bone Morphogenetic Protein-2 (rhBMP-2) in high-risk ankle and hindfoot fusions. Foot Ankle Int 30: 597-603.

11. Bibbo C, Haskell MD (2007) Recombinant Bone Morphogenetic Protein-2 (rhBMP-2) in high-risk foot and ankle surgery: techniques and preliminary results of a prospective, intention to treat study. Techniques in Foot \& Ankle Surgery 6: 71-79.

12. DeVries JG, Nguyen M, Berlet GC, Hyer CF (2012) The effect of recombinant bone morphogenetic protien-2 in revision tibiotalocalcaneal arthrodesis: utilization of the retrograde arthrodesis intramedullary nail database. J Foot Ankle Surg 51: 426-432.

13. Giordano A, Galderisi U, Marino IR (2007) From the laboratory bench to the patient's bedside: an update on clinical trials with mesenchymal stem cells. J Cell Physiol 211: 27-35.

14. Rush SM, Hamilton GA, Ackerson LM (2009) Mesenchymal stem cell allograft in revision foot and ankle surgery: a clinical and radiographic analysis. J Foot Ankle Surg 48: 163-169.

15. Scott RT, Hyer CF (2013) Role of cellular allograft containing mesenchymal stem cells in high-risk foot and ankle reconstructions. J Foot Ankle Surg 52: 32-35.

16. Rush SM (2010) Trinity Evolution: mesenchymal stem cell allografting in foot and ankle surgery. Foot Ankle Spec 3: 140-143.

17. Advanced Biologics (2010) RPT-000327 Report on growth factor concentration in bone and bone marrow aspirate. Data from a single lot of OsteoAMP sponge product, Advanced Biologics, LLC, New Hope, PA, USA.
18. Yeung C, Field J, Roh J (2014) Clinical validation of allogeneic morphogenetic protein: donor intervariability, terminal irradiation and age of product is not clinically relevant. J Spine 3: 173-179.

19. Field J, Yeung C, Roh J (2014) Clinical evaluation of allogeneic growth factor in cervical spine fusion. J Spine 3: 158-160.

20. Roh JS, Yeung CA, Field JS, McClellan RT (2013) Allogeneic morphogenetic protein vs. recombinant human bone morphogenetic protein-2 in lumbar interbody fusion procedures: a radiographic and economic analysis. J Orthop Surg and Res 8: 49

21. Hyer CF, Berlet GC, Bussewitz BW, Hankins T, Ziegler HL, et al. (2013) Quantitative assessment of the yield of osteoblastic connective tissue progenitors in bone marrow aspirate from the iliac crest, tibia, and calcaneus. $J$ Bone Joint Surg Am 95: 1312-1316.

22. Zwingenberger S, Nich C, Valladares RD, Yao Z, Stiehler M, et al. (2012) Recommendations and considerations for the use of biologics in orthopedic surgery. Bio Drugs 26: 245-256.

23. Herkowitz HN, Kurz LT (1991) Degenerative lumbar spondylolisthesis with spinal stenosis. A prospective study comparing decompression with decompression and intertransverse process arthrodesis. J Bone Joint Surg Am 73: 802-808.

24. Zdeblick TA (1993) A prospective, randomized study of lumbar fusion. Preliminary results. Spine (Phila Pa 1976) 18: 983-991.

25. Mankovecky MR, Prissel MA, Roukis TS (2013) Incidence of nonunion of first metatarsal-phalangeal joint arthrodesis with autogenous iliac crest bone graft after failed Keller-Brandes arthroplasty: a systematic review. Journal of Foot and Ankle Surgery 52: 53-55.

26. Dolan CM, Henning JA, Anderson JG, Bohay DR, Kornmesser MJ, et al. (2007) Randomized prospective study comparing tri-cortical iliac crest autograft to allograft in the lateral column lengthening component for operative correction of adult acquired flatfoot deformity. Foot Ankle Int 28: 8-12.

27. Amendola A, Lammens $P$ (1996) Subtalar arthrodesis using interposition iliac crest bone graft after calcaneal fracture. Foot Ankle Int 17: 608-614.

28. Summers BN, Eisenstein SM (1989) Donor site pain from the ilium. A complication of lumbar spine fusion. J Bone Joint Surg $\mathrm{Br}$ 71: 677-680.

29. DeOrio JK, Farber DC (2005) Morbidity associated with anterior iliac crest bone grafting in foot and ankle surgery. Foot Ankle Int 26: 147-151.

30. Schulhofer SD, Oloff LM (1997) Iliac crest donor site morbidity in foot and ankle surgery. J Foot Ankle Surg 36: 155-158.

31. Park JJ, Hershman SH, Kim YH (2013) Updates in the use of bone grafts in the lumbar spine. Bull Hosp Jt Dis (2013) 71: 39-48.

32. Lundh A, Sismondo S, Lexchin J, Busuioc OA, Bero L (2012) Industry sponsorship and research outcome. Cochrane Database Syst Rev 12: MR000033.

33. Roukis TS, Hyer CF, Philbin TM, Berlet GC, Lee TH (2009) Complications associated with autogenous bone marrow aspirate harvest from the lower extremity: an observational cohort study. J Foot Ankle Surg 48: 668-671.

34. Hyer CF, Berlet GC, Bussewitz BW, Hankins T, Ziegler HL, et al. (2013) Quantitative assessment of the yield of osteoblastic connective tissue progenitors in bone marrow aspirate from the iliac crest, tibia, and calcaneus. J Bone Joint Surg Am 95: 1312-1316.

35. McAlister JE, Hyer CF, Berlet GC, Collins CL (2015) Effect of Osteogenic Progenitor Cell Concentration on the Incidence of Foot and Ankle Fusion. J Foot Ankle Surg 54: 888-891.

36. Fu R, Selph S, McDonagh M, Peterson K, Tiwari A, et al. (2013) Effectiveness and harms of recombinant human bone morphogenetic protein-2 in spine fusion: a systematic review and meta-analysis. Ann Intern Med 158: 890-902. 